\title{
CORRECTION
}

\section{Correction to: Intraoperative indocyanine green fluorescence imaging in breast surgery}

\author{
Friedrich Kühn ${ }^{1}$ (D) . Jens-Uwe Blohmer ${ }^{1} \cdot$ Maria Margarete Karsten $^{1}$
}

Published online: 24 June 2020

๑) Springer-Verlag GmbH Germany, part of Springer Nature 2020

\section{Correction to: Archives of Gynecology and Obstetrics https://doi.org/10.1007/s00404-020-05582-7}

In the original article published, some of the superscript characters in Table 1 (column $p$ value with superscript references to the footnotes) were incorrectly inserted by the typesetters. The correct table is given below.

Table 1 General patient data, medical history and surgery

\begin{tabular}{|c|c|c|c|}
\hline Characteristics & $\operatorname{SPY}(n=36)$ & Control $(n=32)$ & $p$ value \\
\hline Age (years) & & & $0.245^{+}$ \\
\hline \multirow[t]{2}{*}{ Mean (SD) } & $43.4(10.7)$ & $46.5(10.7)$ & \\
\hline & & & $0.808^{*}$ \\
\hline$<45$ & $16(44.4 \%)$ & $16(50.0 \%)$ & \\
\hline$\geq 45$ & $20(55.6 \%)$ & $16(50.0 \%)$ & \\
\hline $\mathrm{BMI}\left(\mathrm{kg} / \mathrm{m}^{2}\right)$ & & & $0.280^{\$}$ \\
\hline \multirow[t]{2}{*}{ Median (IQR) } & $23.4(21.3-26.1)$ & $22.1(20.8-26.5)$ & \\
\hline & & & $0.585^{*}$ \\
\hline$<25$ & $20(64.5 \%)$ & $21(72.4 \%)$ & \\
\hline$\geq 25$ & $11(35.5 \%)$ & $8(27.6 \%)$ & \\
\hline Missing & 5 & 3 & \\
\hline Pregnancies & & & $0.197^{\S}$ \\
\hline None & $8(22.2 \%)$ & $8(25.8 \%)$ & \\
\hline 1 & $12(33.3 \%)$ & $11(35.5 \%)$ & \\
\hline 2 & $7(19.4 \%)$ & $10(32.3 \%)$ & \\
\hline$\geq 3$ & $9(25.0 \%)$ & $2(6.5 \%)$ & \\
\hline Missing & - & 1 & \\
\hline Genetic predisposition for breast cancer & & & $0.460^{\S}$ \\
\hline None known & $22(61.1 \%)$ & $17(53.1 \%)$ & \\
\hline BRCA-1/-2 & $13(36.1 \%)$ & $15(46.9 \%)$ & \\
\hline
\end{tabular}

The original article can be found online at https://doi.org/10.1007/ s00404-020-05582-7.

Friedrich Kühn

friedrich.kuehn@charite.de

1 Department of Gynecology, Charité - Universitätsmedizin

Berlin, Charitéplatz 1, 10117 Berlin, Germany 
Table 1 (continued)

\begin{tabular}{|c|c|c|c|}
\hline Characteristics & $\mathrm{SPY}(n=36)$ & Control $(n=32)$ & $p$ value \\
\hline CHEK2 & $1(2.8 \%)$ & $0(0.0 \%)$ & \\
\hline \multicolumn{4}{|l|}{ Comorbidities } \\
\hline Diabetes mellitus & $0(0.0 \%)$ & $1(3.1 \%)$ & $0.478 *$ \\
\hline Missing & 1 & - & \\
\hline Arterial hypertension & $5(14.3 \%)$ & $2(6.3 \%)$ & $0.431 *$ \\
\hline Missing & 1 & - & \\
\hline Smoker & $4(11.1 \%)$ & $5(16.1 \%)$ & $0.723 *$ \\
\hline Missing & - & 1 & \\
\hline Former smoker & $8(22.2 \%)$ & $3(9.7 \%)$ & $0.202 *$ \\
\hline Missing & - & 1 & \\
\hline Prior surgery ${ }^{\mathrm{a}}$ & & & $0.808 *$ \\
\hline $\mathrm{N}(\%)$ & $13(36.1 \%)$ & $12.5(39.1 \%)$ & \\
\hline Type of prior surgery ${ }^{a}$ & & & $0.180^{\S}$ \\
\hline $\mathrm{BCS}$ & $6.5(50.0 \%)$ & $8.5(68.0 \%)$ & \\
\hline Mastectomy & $5(38.5 \%)$ & $1(8.0 \%)$ & \\
\hline Other & $1.5(11.5 \%)$ & $3(24.0 \%)$ & \\
\hline \multicolumn{4}{|l|}{ Local cancer therapy ${ }^{a}$} \\
\hline Surgery & $9.5(73.1 \%)$ & $9.5(76.0 \%)$ & $1.0^{*}$ \\
\hline Adjuvant radiation & $6(46.2 \%)$ & $3(24.0 \%)$ & $0.370 *$ \\
\hline Surgery indication & & & $0.610^{\S}$ \\
\hline Carcinoma & $23(63.9 \%)$ & $24(75.0 \%)$ & \\
\hline Prevention & $10(27.8 \%)$ & $6(18.8 \%)$ & \\
\hline Other & $3(8.3 \%)$ & $2(6.3 \%)$ & \\
\hline Side of surgery & & & $1.0^{*}$ \\
\hline Unilateral & $22(61.1 \%)$ & $20(62.5 \%)$ & \\
\hline Bilateral & $14(38.9 \%)$ & $12(37.5 \%)$ & \\
\hline Type of surgery ${ }^{a}$ & & & $0.275^{\S}$ \\
\hline NSM & $18.5(51.4 \%)$ & $19(59.4 \%)$ & \\
\hline SSM & $10(27.8 \%)$ & $4(12.5 \%)$ & \\
\hline SSM with NAC reconstruction & $2(5.6 \%)$ & $5(15.6 \%)$ & \\
\hline Other & $5.5(15.3 \%)$ & $4(12.5 \%)$ & \\
\hline Neoadjuvant cancer therapy & & & $0.244 *$ \\
\hline $\mathrm{N}(\%)$ & $23(63.9 \%)$ & $24(75.0 \%)$ & \\
\hline Chemotherapy & $8(34.8 \%)$ & $13(54.2 \%)$ & \\
\hline None & $15(65.2 \%)$ & $11(45.8 \%)$ & \\
\hline Duration of surgery (h) & & & $0.124^{+}$ \\
\hline Mean (SD) & $2: 43(0: 48)$ & $2: 24(0: 52)$ & \\
\hline Resection weight $(\mathrm{g})^{\mathrm{a}}$ & & & $0.153^{\$}$ \\
\hline Median (IQR) & $260(149.3-381.8)$ & $254.5(173-449.3)$ & \\
\hline & & & $0.692 *$ \\
\hline$<500 \mathrm{~g}$ & $33.5(93.1 \%)$ & $25.5(86.4 \%)$ & \\
\hline$\geq 500 \mathrm{~g}$ & $2.5(6.9 \%)$ & $4(13.6 \%)$ & \\
\hline Missing & - & 2.5 & \\
\hline Implant insertion ${ }^{\mathrm{a}}$ & & & $0.197 *$ \\
\hline $\mathrm{N}(\%)$ & $28.5(79.2 \%)$ & $29.5(92.2 \%)$ & \\
\hline Implant shape ${ }^{a}$ & & & $0.023 *$ \\
\hline Round & $0(0.0 \%)$ & $5.5(19.3 \%)$ & \\
\hline Anatomic & $27.5(100 \%)$ & $23(80.7 \%)$ & \\
\hline
\end{tabular}


Table 1 (continued)

\begin{tabular}{|c|c|c|c|}
\hline Characteristics & SPY $(n=36)$ & Control $(n=32)$ & $p$ value \\
\hline Missing & 1 & 1 & \\
\hline Implant position $^{\mathrm{a}}$ & & & $0.070^{*}$ \\
\hline Epimuscular & $11(38.6 \%)$ & $18.5(62.7 \%)$ & \\
\hline Submuscular & $17.5(61.4 \%)$ & $11(37.3 \%)$ & \\
\hline Implant volume $(\mathrm{ml})^{\mathrm{a}}$ & & & $0.398^{\$}$ \\
\hline Median (IQR) & $331.3(257.5-420)$ & $308.8(185-448.1)$ & \\
\hline Expander insertion $^{\mathrm{a}}$ & & & $0.434 *$ \\
\hline $\mathrm{N}(\%)$ & $5(13.9 \%)$ & $2(6.3 \%)$ & \\
\hline Expander shape $^{a}$ & & & - \\
\hline Round & $0(0.0 \%)$ & $0(0.0 \%)$ & \\
\hline Anatomic & $5(100 \%)$ & $2(100 \%)$ & \\
\hline Expander position $^{\mathrm{a}}$ & & & $1.0^{*}$ \\
\hline Epimuscular & $2(40.0 \%)$ & $1(50.0 \%)$ & \\
\hline Submuscular & $3(60.0 \%)$ & $1(50.0 \%)$ & \\
\hline Expander volume $(\mathrm{ml})^{\mathrm{a}}$ & & & $0.833^{\$}$ \\
\hline Median (IQR) & $50(50-225)$ & $155(-)$ & \\
\hline Autologous reconstruction (TRAM) ${ }^{\mathrm{a}}$ & $2(5.6 \%)$ & $0(0.0 \%)$ & $0.494 *$ \\
\hline Mesh insertion ${ }^{\mathrm{a}}$ & $11(30.6 \%)$ & $6(18.8 \%)$ & $0.401 *$ \\
\hline $\mathrm{ADM}$ insertion $^{\mathrm{a}}$ & $4(11.1 \%)$ & $1(3.1 \%)$ & $0.360 *$ \\
\hline Lymph node intervention ${ }^{a}$ & & & $0.537^{\S}$ \\
\hline $\mathrm{N}(\%)$ & $18.5(51.7 \%)$ & $16(50.0 \%)$ & \\
\hline SNB & $15.5(83.8 \%)$ & $13(81.3 \%)$ & \\
\hline AS & $2(10.8 \%)$ & $3(18.8 \%)$ & \\
\hline $\mathrm{AD}$ & $1(5.4 \%)$ & $0(0.0 \%)$ & \\
\hline \multicolumn{4}{|c|}{ Subset with reduced perfusion in ICG fluorescence angiography ${ }^{\mathrm{a}}$} \\
\hline $\mathrm{N}(\%)$ & $11.5(31.9 \%)$ & - & \\
\hline Resection of tissue with reduced perfusion ${ }^{a}$ & & - & \\
\hline Yes & $4(34.8 \%)$ & - & \\
\hline No & $7.5(65.2 \%)$ & - & \\
\hline
\end{tabular}

Numbers in italics are missing values

$B C S$ Breast conserving surgery, NSM nipple sparing mastectomy, SSM skin-sparing mastectomy, NAC nipple areola complex, SNB sentinel lymph node biopsy, $A S$ axillary sampling, $A D$ axillary dissection, $I C G$ indocyanine green, $A D M$ acellular dermal matrix, $S D$ standard deviation, $I Q R$ interquartile range

${ }^{a}$ In the calculation of breast specific parameters women who underwent bilateral surgery were only counted as half item for each breast which ensures that no information gets lost and that no patient is counted twice in any category

Test on normal distribution: Shapiro-Wilk test

+ t-test for independent samples

$\$$ Mann-Whitney- $U$ test

*Fisher's exact test

$\S$ Chi-square-test

Publisher's Note Springer Nature remains neutral with regard to jurisdictional claims in published maps and institutional affiliations. 